\title{
THE IMPROVEMENT OF ATOMIC FORCE MICROSCOPE SUITABLE FOR MAGNETIC DOMAIN STRUCTURE MEASUREMENTS
}

\section{Sveklo, W. Dobrogowski, M. Kisielewski and A. MaziewsKi}

Institute of Physics of Białystok University, Lipowa 41, 15-424 Białystok, Poland

Based on commercial Burleigh METRIS ${ }^{T M}-2000$ Atomic Force Microscope two methods of magnetic force measurements were realised. The developed system was successfully applied for study of magnetic structure of both YIG-garnet and $\mathrm{CoNi} / \mathrm{Pt}$ magneto-optic multilayers.

PACS numbers: 75.70.-i, 75.30.Pd, 61.16. Ch

At the end of eighties, the atomic force microscopy (AFM) was for the first time applied for investigation of a magnetic stray field. At that moment a novel, very powerful experimental technique predestined for magnetisation spatial distribution study, named magnetic force microscopy (MFM), was born and has been up to now intensively developed. Magnetic domain structures could be also studied using many other techniques, such as for example: magneto-optical Kerr and Faraday microscopy, Bitter pattern technique, ferrofluid decoration, cryogenic condensation, Lorentz microscopy, electron holography, and scanning electron microscopy with polarisation analysis (SEMPA). A short overlook of these techniques and their advantage and disadvantage in respect of MFM can be found in Ref. [1]. Although there is a wide offer of commercial MFM today, a significant number of nowadays working MFMs was home-built. Improvement of a "low-cost" AFM microscope for MFM investigation is the main purpose of our work. We have focused our efforts on commercial Burleigh METRISTM-2000 Atomic Force Microscope.

MFM allows to obtain magnetic structure images with resolution greater than that one achieved by optical methods. The principal scheme of MFM is the further development of AFM. In comparison with the forces typically used for AFM work, magnetic forces are significantly smaller in value and their registration requires notable efforts. Due to the low gradient of magnetic field over the sample surface, it is impossible to use its action for feedback control and, consequently, during magnetic field measurements the feedback loop must be broken.

In the case of our AFM, a standard working algorithm, which allows magnetic measurements, is discussed in Ref. [2]. It could be briefly described as follows: At the beginning magnetic probe is far away from the surface of the sample, feedback is on; then the probe approaches to the sample. Further feedback is 


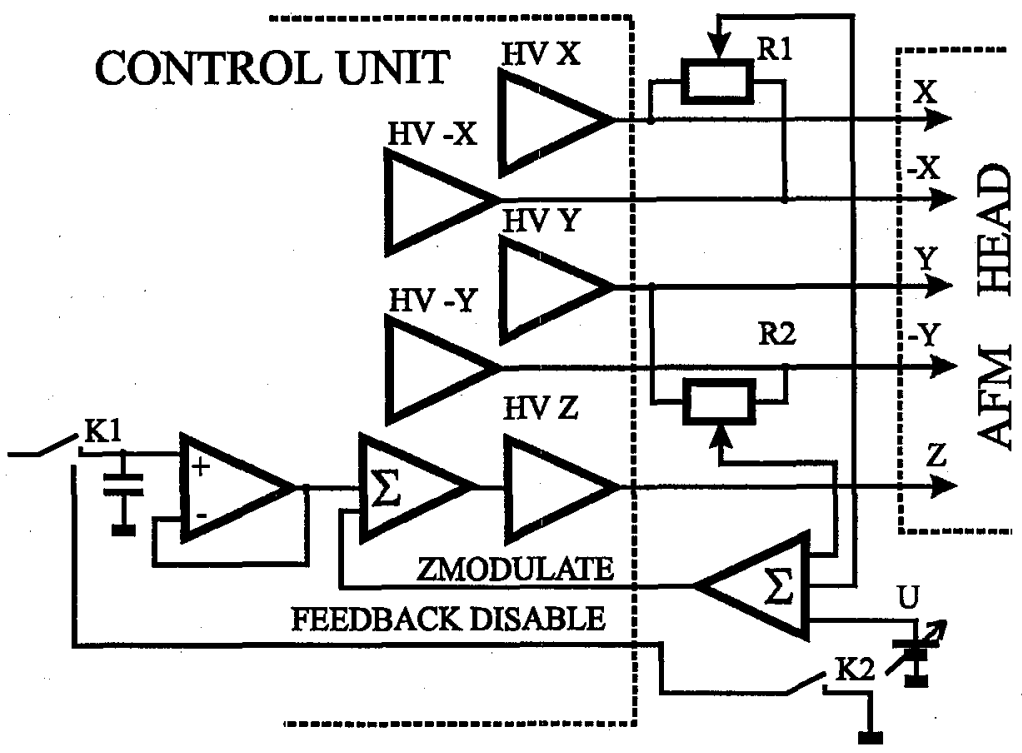

Fig. 1. The scheme of the Burleigh METRIS ${ }^{\text {TM }}-2000$ AFM modernisation for MFM measurements. External resistors $R 1$ and $R 2$ allow to add an additional voltage to $Z$ channel proportional to the output signal of high voltage (HV) $X, Y$ amplifiers during scanning, which compensates the sample-scanning plane inclination. The $U$ variable voltage source is used for probe sample separation, switch $K 2-$ for feedback turning on/off.

broken and additional voltage is supplied to channel $Z$ to retract the probe from the surface and overcome adhesion force. After this, the probe must be scanned (in $X Y$-plane) at a constant distance from the surface and then the cantilever displacement, caused by the action of the magnetic stray field of the sample on the magnetic probe, is measured. To obtain maximum sensitivity and exclude the influence of shorter-range van der Waals forces, the probe must be scanned at a stable distance of about $50 \mathrm{~nm}$ over the sample surface. Roughness of sample surface and its inclination to the scanning plane will lead to an undesirable change of the probe-sample distance, so these two factors must be accounted for during scanning.

Investigations were performed for the correction of the roughness of sample surface and its inclination to the scanning plane and - as a result - two methods were realised. The commercial AFM silicon tip (cantilever spring constant $1 \mathrm{~N} / \mathrm{m}$ ) with deposited Co-Pt film and magnetised in $5 \mathrm{kOe}$ field was used as magnetic probe.

A simple electronic compensation of sample inclination was used in the first method (Fig. 1). After the probe was approached to the sample, the $K 2$ switch was used for turning off the feedback. Next, using additional variable voltage source $U$, the probe was separated from the sample surface. Then, during the sample scanning, such states of resistors $R 1$ and $R 2$ have been found that the probe no more 
clung to the sample surface, i.e. the sample surface was parallel to the scanning plane. Finally, the magnetic image could be obtained using the standard AFM scanning procedure (force is registered). To increase the signal-to-noise ratio for MFM measurements, it is recommended to make multiple signal storage in a point. Usually, during AFM imaging, one has a great signal and additional point storage is not necessary. After modifying the supplied measuring program we obtain the possibility of introducing additional data storage. In Fig. 2 the raw MFM images of the same surface of the CAMST-2 reference sample (CoNi/Pt magneto-optic multilayers) [3], obtained using the first method with different storage number in a point, are submitted.
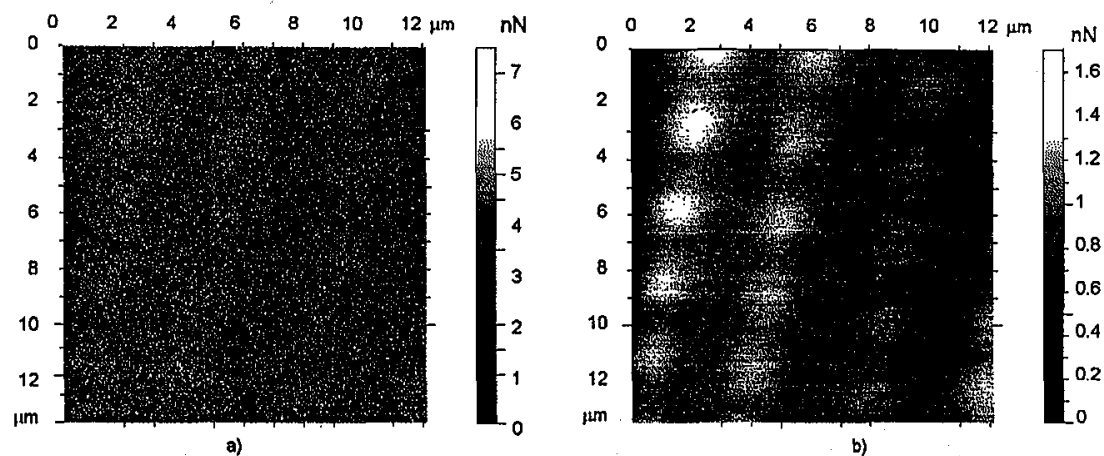

Fig. 2. Raw AFM-images of magnetic structure of CAMST-2 reference sample with different data storage in the point: (a) 1, (b) 64 .

In many cases the roughness of the sample demands more complicated local correction of the sample-probe distance. Such correction was realised in our second method, which is similar to the "lift-mode" method, introduced by Digital Instruments and applied in its commercial MFMs. The second method requires significant change of the scanning algorithm. All control functions are provided by computer, equipped with additional digital-to-analog converter (DAC) for modulation of the $Z$ canal and one digital output canal for the feedback control. At first the sample is scanned in a standard contact AFM mode to obtain a typical surface topographic map. In the next step the feedback is broken, the probe is moved up at required distance and the scanning is put in motion once again. During the second, cardinal scanning the distance between sample and probe is driven to be constant for each imaged point. It is done by the modification of the position of the probe along the $Z$ coordinate, according to the previously obtained topographic data.

A comparison of these two methods showed that in the case of a smooth plane sample surface they both give the same result. A significant advantage of the second method reveals in the case of rough sample surface (Fig. 3). As a main difficulty of the second method realisation one can point the necessity of writing a completely new computer program for the MFM measurements.

As a further example of the efficacy of the developed method, the domain structure of magnetic soft YIG-garnet with out-of-plane magnetisation is showed 

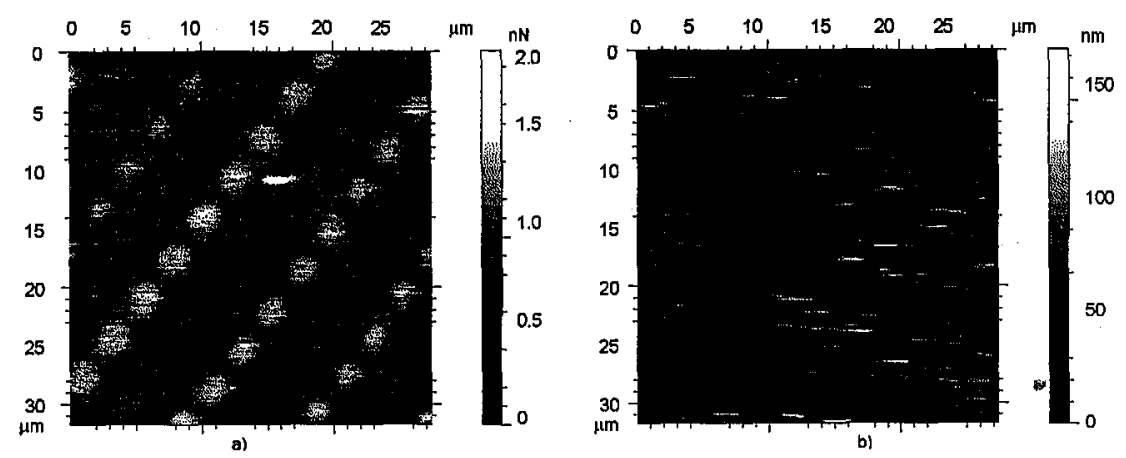

Fig. 3. (a) Raw MFM image of CAMST-2 reference sample [3] measured with "lift mode" method. Data storage in a point 1024. (b) AFM image of the same region. Plane inclination $0.95 \mu \mathrm{m}$ is subtracted.
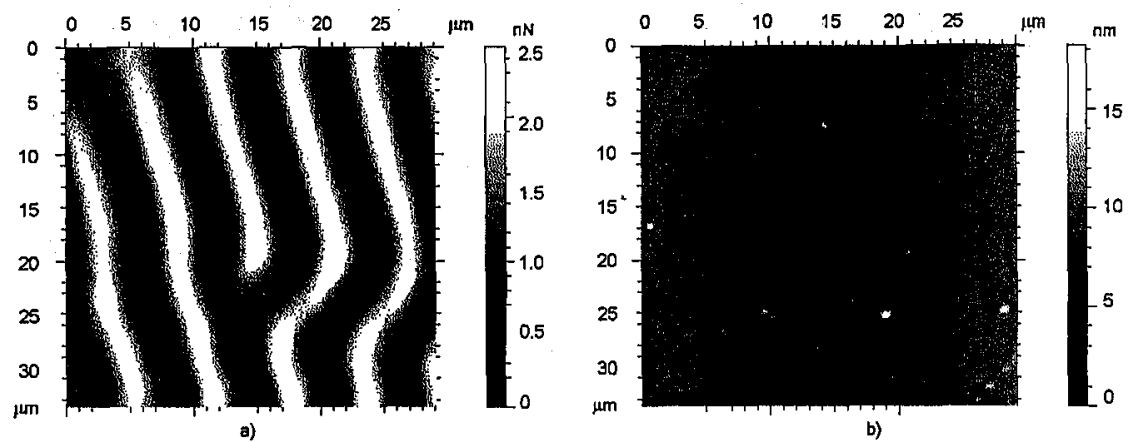

Fig. 4. (a) Domain structure of YIG sample (film thickness $14 \mu \mathrm{m}$ ) with out-of-plane magnetisation measured with "lift mode" method. (b) AFM image of the same region. Plane inclination $0.2 \mu \mathrm{m}$ is subtracted.

in Fig. 4. For comparison the topographic image of the same place of the sample is presented, too. The use of the multiple data storage in a point, allows us tightly approach to the noise limit caused by temperature fluctuation of cantilever deflection (for spring constant $1 \mathrm{~N} / \mathrm{m}$ the fluctuation is in the range of $\approx 10^{-10} \mathrm{~N}$ ). But main shortcoming of MFM measurements is connected with smooth magnetic field distribution outside the sample.

This work was partially supported by ESF NANOMAG Programme and Polish Grant No. 2P03B06515.

\section{References}

[1] R. Wiesendanger, H.J. Guntherodt, Scanning Tunneling Microscopy II, Springer-Verlag, Berlin 1992.

[2] C.D. Wright, Surface Topography Applications, Vol. 5, Rev. B 2/98, Burleigh Instruments 1998.

[3] Information Storage technology group of the MESA institute of the University of Twente (http://www.el.utwente.nl/tdm/istg/index.html). 